\title{
VARIABILIDADE ESPACIAL DO POTENCIAL de ERosão das CHUVAS do estado de SÃo PaUlo $\left(^{1}\right.$ )
}

\author{
SIDNEY ROSA VIEIRA $\left({ }^{2,3}\right)$ e FRANCISCO LOMBARDI NETO $\left.{ }^{2}\right)$
}

\begin{abstract}
RESUMO
Utilizaram-se dados de 292 pluviômetros da rede oficial do Estado de São Paulo para o cálculo do fator $\mathbf{R}$ da equação universal de perdas de solo, segundo modelo disponível na literatura. O objetivo deste trabalho foi usar a geoestatística para estudar a variabilidade espacial do potencial de erosão das chuvas e construir mapas de isolinhas com precisão. Calculou-se a dependência espacial através do semivariograma para os períodos das secas (abril a setembro), das águas (outubro a março) e para a erosividade média anual. Os alcances dos semivariogramas variaram de 50 a $70 \mathrm{~km}$, porém a autovalidação das estimativas mostrou que o número de vizinhos utilizados em uma estimativa não deve ultrapassar 16 para que as hipóteses a respeito dos dados não sejam invalidadas. Estimou-se um total de 2.460 valores para todo o Estado, com uma separação de $20 \mathrm{~km}$ entre pontos para boa precisão na construção dos mapas de isolinhas. Os mapas de isolinhas mostraram que, no período das secas, existe aumento de erosividade na direção sul do Estado; no período das águas, dá-se o inverso: o aumento da erosividade é na direção norte.
\end{abstract}

Termos de indexação: erosividade, mapeamento, krigagem, conservação do solo.

\section{ABSTRACT \\ SPATIAL VARIABILITY OF RAINFALL EROSIVITY FOR THE STATE OF SÃO PAULO, BRAZIL}

Rainfall data from 292 raingauges operated by the State of São Paulo were used to calculate the rainfall erosivity factor from the Universal Soil Loss Equation using a model available from the literature. The objective of this work was to use geostatistics to study the spatial variability of the rainfall erosivity and to construct isoline maps with high precision. Spatial autocorrelation was calculated

(1) Recebido para publicação em 11 de agosto de 1994 e aceito em 31 de agosto de 1995.

(2) Seção de Conservação do Solo, Instituto Agronômico (IAC), Caixa Postal 28, 13001-970 Campinas (SP).

$\left({ }^{3}\right)$ Com bolsa de pesquisa do CNPq. 
with semivariograms for the rainy (October to March) and dry seasons (April to September), and for the yearly average. The range of the semivariograms were within 50 and 70 kilometers, however, the jack-knifing technique showed that only the 16 nearest neighbors should be used in order to stay within distances where stationarity existed. A total of 2,460 values were estimated on a 20 kilometers grid to allow for a good precision of contour maps. The contour maps showed that during the dry season the erosivity increases southward. During the rainy season, this trend inverts itself, with the erosivity increasing northward.

Index terms: erosivity, mapping, kriging, soil conservation.

\section{INTRODUÇÃO}

Estimativas de perdas de solos podem servir como guia na seleção de sistemas de cultivo e práticas de conservação do solo em geral. Existem vários modelos disponíveis para predição de perdas de solo por erosão, como a equação universal de perdas de solo (EUPS) (Wischmeier \& Smith, 1978); Chemical, Runoff, and Erosion from Agricultural Management System (CREAMS) (Knisel, 1980), e Water Erosion Prediction Project (WEPP) (Flanagan, 1991), porém, no Brasil, a equação universal de perdas de solo ainda tem sido de uso mais generalizado (Bertoni \& Lombardi Neto, 1990). O cálculo das perdas de solo através da equação universal de perdas de solo envolve o conhecimento de cada um de seus fatores $\mathrm{R}$ (erosividade da chuva), K (erodibilidade do solo), $\mathrm{L}$ (comprimento de rampa), S (declividade), C (cobertura) e P (práticas de manejo), para as condições locais, os quais são relativamente difíceis de obter com precisão, em particular o fator R. O cálculo deste fator, utilizando método proposto por Wischmeier \& Smith (1958) é lento e por demais trabalhoso por envolver informações contidas em diagramas diários de chuva, estes, por sua vez, escassos ou inexistentes. Tal método envolve o produto da energia cinética da chuva pela intensidade máxima em 30 minutos, resultando em um valor denominado $\mathrm{EI} 30$.

A média anual do EI30, correspondente a longos períodos (20 anos ou mais), tem sido usada como o fator R da equação universal de perdas de solo. Lombardi Neto e Moldenhauer (1992), utilizando longas séries de dados para Campinas (SP), encontraram alta correlação entre o valor médio mensal do $\mathrm{EI}_{30}$ e o valor médio mensal do coeficiente de chuva $(\mathrm{p} / \mathrm{P})$, sendo $\mathrm{p}$ a precipitação média mensal e $\mathrm{P}$, a precipitação média anual, ambas em $\mathrm{mm}$. Assim, tornou-se possível o cálculo do fator $\mathrm{R}$ sem o uso de diagramas de pluviógrafo.

Uma das maneiras mais comuns e ilustrativas de apresentar mapas de variáveis espaciais é através de isolinhas. Existem, basicamente, três maneiras de preparar os dados para a construção de mapas de isolinhas: (1) medir a variável em estudo no menor espaçamento possível; (2) medir em um espaçamento qualquer e interpolar usando pacotes de programas computacionais comerciais; (3) medir num espaçamento que permita obtenção de dependência espacial e interpolar usando o método da krigagem (Vieira et al., 1981). A primeira é praticamente inviável, haja vista o custo de amostragem e análise. A segunda pode gerar isolinhas completamente diferentes da realidade, uma vez que os métodos utilizados por pacotes comerciais não consideram a variabilidade espacial durante a interpolação (Vieira et al., 1981). A terceira é a ideal porque pode economizar amostragem e interpolar sem tendenciosidade e com mínima variância do erro de interpolação (Burgess \& Webster, 1980). Em situações em que a densidade de amostragem já é estabelecida, como a rede de pluviômetros no Estado de São Paulo, a construção de mapas de isolinhas envolve a verificação da dependência espacial nas amostras existentes para a aplicação do método krigagem. Caso a dependência não exista, outros métodos de interpolação que não utilizam csta propriedade devem ser aplicados. O método da krigagem tem sido extensivamente usado em estudos envolvendo mapas de isolinhas de propriedades do solo (Burgess \& Webster, 1980; Hajrasuliha et al., 1980; Vieira et al., 1981, 1983, 1991). 
O objetivo deste trabalho é utilizar dados obtidos segundo modelo proposto por Lombardi Neto \& Moldenhauer (1992) para estimar a erosividade das chuvas a partir de dados de pluviômetro, estudar sua variabilidade e utilizar o método da krigagem para construção de mapas de isolinhas para o Estado de São Paulo.

\section{MATERIAL E MÉTODOS}

Utilizaram-se totais mensais de 292 locais com 20 anos ou mais, extraídos dos Boletins Pluviométricos do Departamento de Águas e Energia Elétrica (São Paulo, 1973). Esses dados foram digitados em computador com as seguintes informações adicionais: localização do pluviômetro em Iongitude e latitude, altitude $(\mathrm{m})$, valores mensais de precipitação $(\mathrm{mm})$ e total anual de precipitação $(\mathrm{mm})$.

As precipitações pluviais das regiões próximas ao litoral apresentaram um comportamento distinto do restante do Estado em relação à variabilidade espacial, como já havia sido verificado por Vieira et al. (1991). Por essa razão, os dados referentes ao litoral não foram utilizados.

Para cada local e para cada mês, o índice de erosão média mensal $\left(E I_{m}\right)$ pode ser calculado, segundo Lombardi Neto \& Moldenhauer (1992), por:

$$
\mathrm{EI}_{\mathrm{m}}=68,730\left(\mathrm{p}^{2} / \mathrm{P}\right)^{0,841}
$$

onde

p é a precipitação média mensal (mm), e P é a precipitação média anual $(\mathrm{mm})$.

O fator R (MJ.mm/ha.h.ano) da equação universal de perdas de solo, ou seja, a erosividade da chuva para um local, é calculado por:

$$
\mathrm{R}=\sum_{\mathrm{i}=1}^{12} \mathrm{EI}_{\mathrm{m}}
$$

Para os objetivos deste trabalho, além da erosividade anual, dividiu-se o ano em dois períodos: o das secas, de abril a setembro, e o das águas, de outubro a março. A erosividade para esses períodos foi obtida somando-se os valores mensais dos meses correspondentes.

As coordenadas geográficas dos pluviômetros, originalmente expressas em graus de longitude e latitude, foram matematicamente transformadas e expressas em quilometros, a partir de um ponto arbitrário de origem de um sistema cartesiano de coordenadas situado na interseção da latitude $25^{\circ} 30^{\prime} \mathrm{e}$ longitude $53^{\circ} 30^{\prime}$.

A dependência espacial entre valores de erosividade da chuva pode ser calculada pela semivariância, $\gamma(\mathrm{h})$ :

$$
\gamma(h)=\frac{1}{2 N(h)} \sum_{i=1}^{N(h)}\left[Z\left(x_{i}\right)-Z\left(x_{i}+h\right)\right]^{2}
$$

onde

$\mathrm{N}(\mathrm{h})$ é o número de pares de valores $\left[\mathrm{Z}\left(\mathrm{x}_{\mathrm{i}}\right), \mathrm{Z}\left(\mathrm{x}_{\mathrm{i}}\right.\right.$ $+h$ )] separados por um vetor $\mathbf{h}$, e $\mathbf{x}_{\mathbf{i}}$ é uma posição espacial da variável $\mathrm{Z}$, no caso, a erosividade. À nuvem de pontos $[h, \gamma(h)]$ ajusta-se uma função matemática, cujos parâmetros são conhecidos como: efeito pepita, correspondendo ao valor da interseção no eixo das semivariâncias; patamar, aproximadamente igual ao valor variância dos dados, e alcance, que representa a distância na qual o semivariograma atinge o valor do patamar (Vieira et al., 1983). Se a variabilidade espacial ocorrer de modo idêntico, isto é, com o mesmo semivariograma, em todas as direções, então a variável espacial sob estudo ocorre em condições isotrópicas e a direção do vetor $h$ não precisa ser considerada, importando apenas o seu módulo, ou seja, a distância entre as amostras. Isso pode ser verificado através da técnica de autovalidação, conhecida como "jack-knifing", onde se estima um valor conhecido e calculam-se os desvios padronizados, isto é, a diferença entre valores estimados e medidos, dividida pela raiz quadrada da variância da estimativa, para os quais se espera que média e variância devam ser, respectivamente, 0 e 1 , se o modelo do semivariograma foi bem escolhido (Vieira et al., 1983). Quando os valores das semivariâncias aumentam com a distância sem ter limites claros, existe uma tendência de aumento considerável nos 
dados em alguma direção, o que pode invalidar a hipótese de estacionaridade estatística de $2{ }^{a}$ ordem. Se isso acontecer, é necessário remover tal tendência como indicaram Vieira et al. (1992). Os semivariogramas utilizados neste trabalho foram escalonados como sugeriram Vieira et al. (1991), para facilitar sua comparação. Para o escalonamento, dividem-se os semivariogramas de cada variável espacial pelas respectivas variâncias. Se os valores de $\gamma(h)$ forem crescentes, até em alguma distância na qual se estabiliza, então, dependência e estacionaridade existem, e podem ser usadas na estimativa de valores para locais $\mathrm{x}_{0}$, onde a propriedade não foi medida, através da equação:

$$
\mathrm{Z}^{*}\left(\mathrm{x}_{0}\right)=\sum_{\mathrm{i}=1}^{\mathrm{N}} \lambda_{\mathrm{i}} \mathrm{Z}\left(\mathrm{x}_{\mathrm{i}}\right)
$$

onde

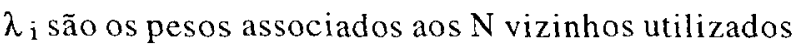
na estimativa.

Quando a equação (4) é submetida às condições de não-tendenciosidade e de mínima variância do erro de interpolação, cuja minimização é feita através do processo do multiplicador de Lagrange, chega-se ao sistema da krigagem:

$$
\begin{aligned}
& \sum_{j=1}^{N} \lambda j \gamma\left(x_{i}, x_{j}\right)+\mu=\gamma\left(x_{i}, x_{0}\right), i=1, N \\
& \sum_{j=1}^{N} \lambda j=1
\end{aligned}
$$

cuja solução gera $\mathrm{N}$ pesos, $\lambda \mathrm{i}$, um multiplicador de Lagrange, $\mu$, e possibilita o cálculo da equação (4) e também a variância da estimativa, através da equação:

$$
\sigma_{k}^{2}\left(X_{0}\right)=\sum_{j=1}^{N} \lambda_{j} \gamma\left(x_{j}, x_{0}\right)+\mu
$$

Assim, podem-se estimar valores da erosividade, para qualquer posição no Estado e, também, saber qual a confiança correspondente a tal estimativa.
Dessa maneira, evita-se que estimativas sejam feitas por processos desconhecidos para todos os locais na área em estudo.

Utilizando-se valores estimados através da krigagem em espaçamentos regulares, pode-se construir mapas de isolinhas com alta precisão e com variância conhecida. Isso é possível, uma vez que, dentro da escala original dos dados, qualquer que seja o método de construção de isolinhas, basear-se-á em uma densidade maior de pontos. A variância do erro das estimativas é automaticamente calculada nas interpolações pela krigagem, mediante a equaçāo (5). Estabeleceu-se um reticulado quadrado de $20 \mathrm{~km}$ de espaçamento entre pontos para as estimativas, totalizando 2.460 pontos dentro dos limites do Estado de São Paulo. Os programas computacionais de geoestatística utilizados neste trabalho foram desenvolvidos por Vieira et al. (1983) e aperfeiçoados por Vieira et al. $\left({ }^{4}\right)$. Os mapas de isolinhas foram construídos usando 2.460 valores estimados em um programa comercial denominado SURFER.

\section{RESULTADOS E DISCUSSÃO}

Os principais parâmetros estatísticos para as erosividades correspondentes aos períodos das secas, das águas e anual são mostrados no quadro 1. Os coeficientes de simetria e de curtose das distribuições empíricas de freqüência e calculados para os períodos de total anual, das águas e das secas, são mostrados para efeito comparativo com a distribuição normal, para a qual estes coeficientes são, respectivamente, 0 (zero) e 3 (três). Não é objetivo deste trabalho verificar a distribuição de freqüências exatas das erosividades, a qual seria efetuada com testes estatísticos específicos para este fim. Todavia, a comparação dos coeficientes de simetria e de curtose para as erosividades com os valores da distribuição normal, embora subjetiva, permite saber se existe uma tendência de aproximação da distribuição log normal, para a qual os valores de simetria são maiores do que 3 .

$\left(^{4}\right)$ VIEIRA, S.R.; MILLETE, J.A.; REYNOLDS, W.D. \& TOPP, G.C. Handbook on the use of geostatistics in spatial variability studies. Agricultural Canada Bulletin, 1995. (Enviado para publicação) 
Assim, aparentemente, apenas a erosividade correspondente ao período das águas se aproxima da distribuição normal. Dados cujos coeficientes de simetria e curtose são da ordem daqueles do período das secas têm, provavelmente, distribuição log normal, e seria ideal trabalhar com os logaritmos.

Para evitar a mistura de distribuições, porém, uma vez que a erosividade correspondente ao período das águas se aproxima da distribuição normal, todos foram mantidos sem nenhuma transformação. Deve-se lembrar que os cálculos usados em geoestatística não requerem o conhecimento da distribuição de freqüências da variável. Os coeficientes de variaçāo tendem a aumentar à medida que a erosividade diminui, com valor máximo para o período das secas.

É importante ressaltar a grande diferença entre os valores mínimo e máximo no quadro 1, principalmente para o período das secas. Isso mostra que, no período aqui arbitrariamente agrupado como das secas, existe uma variabilidade muito grande, com locais onde chove muito pouco e locais onde há chuvas suficientes para causar uma erosividade calculada de $1.424 \mathrm{MJ} . \mathrm{mm} / \mathrm{ha}$.h.

Os semivariogramas para as erosividades correspondentes ao período das águas (outubro a março) e para o anual estão na figura 1, onde a linha contínua representa o modelo ajustado, enquanto o esférico ajustado segue a equação:

$$
\begin{aligned}
& \gamma(h)=0,1+0,9\left[1,5\left(\frac{h}{70}\right)-0,5\left(\frac{h}{70}\right)^{3}\right] ; h \leq 70 \\
& \gamma(h)=0,1+0,9 \quad h>70
\end{aligned}
$$

Informações mais detalhadas sobre modelos para semivariogramas e técnicas para ajuste podem ser encontradas em Vieira et al. (1983). De modo geral, pode-se dizer que o parâmetro efeito pepita de 0,1 indica boa continuidade entre valores vizinhos. $\mathrm{O}$ alcance de $70 \mathrm{~km}$ garante que todos os vizinhos localizados dentro de um círculo com este raio são tão parecidos uns com os outros que podem ser usados para estimar valores para qualquer ponto entre eles. A existência de um patamar indica que a estacionaridade está garantida. Um fato bastante importante a ser notado na figura 1 é que os semivariogramas para o período das águas e total anual, quando escalonados, sobrepuseram-se quase perfeitamente. Isso indica que as suas variabilidades espaciais são parecidas. Indica, também, que a contribuição do período das secas para a erosividade anual é pequena e não muda, significativamente, sua variação espacial.

A figura 2 mostra o semivariograma para erosividade correspondente ao período das secas. O modelo ajustado foi o exponencial com a equação:

$$
\gamma(h)=0,2+0,8\left[1-\operatorname{EXP}\left(\frac{-3 h}{50}\right)\right]
$$

Tal semivariograma foi calculado, na realidade, para os resíduos da tendência parabólica ajustada aos dados pela equação:

$$
\begin{aligned}
Z_{\text {est }}= & 3.323-3,784 \mathrm{x}-7,100 \mathrm{y}+ \\
& +0,0175 \mathrm{x}^{2}+0,0337 \mathrm{y}^{2}+0,045 \mathrm{x} \cdot \mathrm{y}
\end{aligned}
$$

cujo coeficiente de correlação foi de 0,7422 . A remoção da tendenciosidade foi necessária porque o semivariograma para os dados originais apresentou crescimento sem limites com a distância. Pelo

Quadro 1. Estatísticas da erosividade (R) para o período anual das águas e das secas

Erosividade, MJ.mm/ha.h.ano

\begin{tabular}{lrrrrrrr} 
Período & Média & Variância & C.V. & Mínimo & Máximo & Simetria & Curtose \\
\hline \multirow{2}{*nnnnnyyy}{ Anual } & 6632 & 564500 & 11,33 & 5262 & 9765 & 0,9085 & 4,064 \\
Águas & 6002 & 757000 & 14,50 & 4035 & 9048 & 0,5047 & 3,230 \\
Secas & 630 & 44980 & 33,65 & 239 & 1424 & 1,0570 & 4,266 \\
\hline
\end{tabular}


valor do efeito pepita, 0,2 , pode-se deduzir que existe uma variação ao acaso maior para esse período do que para o das águas. Isto corrobora os dados do quadro 1 , os quais revelam um coeficiente de variação para o período das secas muito maior do que para o das águas, ou para o anual.

A figura 3 mostra o mapa de isolinhas médias para a erosividade anual com isolinhas de 5.500 até $8.250 \mathrm{com}$ intervalo de $250 \mathrm{MJ} . \mathrm{mm} / \mathrm{ha}$.h. Em geral, nota-se leve crescimento dos valores das isolinhas na direção sul-norte, portanto, com os menores valores nas regiões próximas do Paraná, crescendo até um máximo nas regiões próximas ao oeste de Minas Gerais. Com isso, a região Nordeste do Estado tem valores de erosividade anual de 7.500 a 8.250 $\mathrm{MJ} . \mathrm{mm} / \mathrm{ha}$.h. Isso deve ser levado em conta em planejamento conservacionista para tais regiões. Nota-se, também, grande aumento nos valores de erosividade na região da Serra da Mantiqueira, devido ao aumento da precipitação nas regiões de maior altitude.

Apesar de existirem indícios de paralelismo na distribuição das isolinhas na direção leste-oeste, para a crosividade anual - Figura 3 - eles não foram suficientes para invalidar as hipóteses de estacionaridade, como pode ser visto pelo semivariograma com patamar bastante nítido - Figura 1 - conforme também indicaram Vieira et al. (1992).

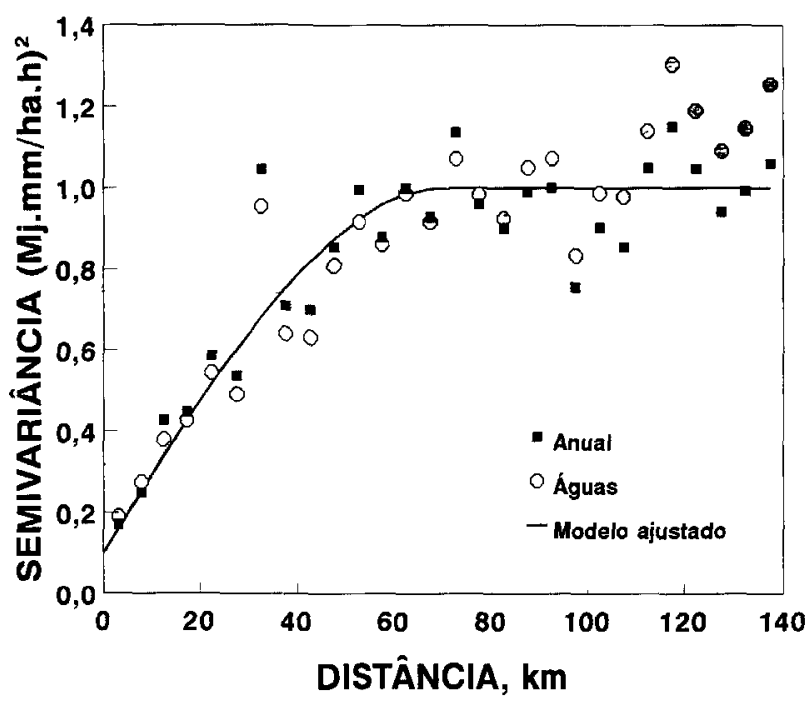

Figura 1. Semivariograma escalonado para a erosividact anual e para a do período das águas, com modelo esférico ajustado
A figura 4 mostra o mapa de isolinhas para o período das águas. Com exceção dos valores absolutos das erosividades, é bastante semelhante ao da erosividade anual - Figura 3. Este fato confirma o bom escalonamento para os semivariogramas correspondentes - Figura 1 . Pode-se dizer, então, que a variabilidade espacial da erosividade correspondente ao período das águas define a variabilidade da erosividade anual.

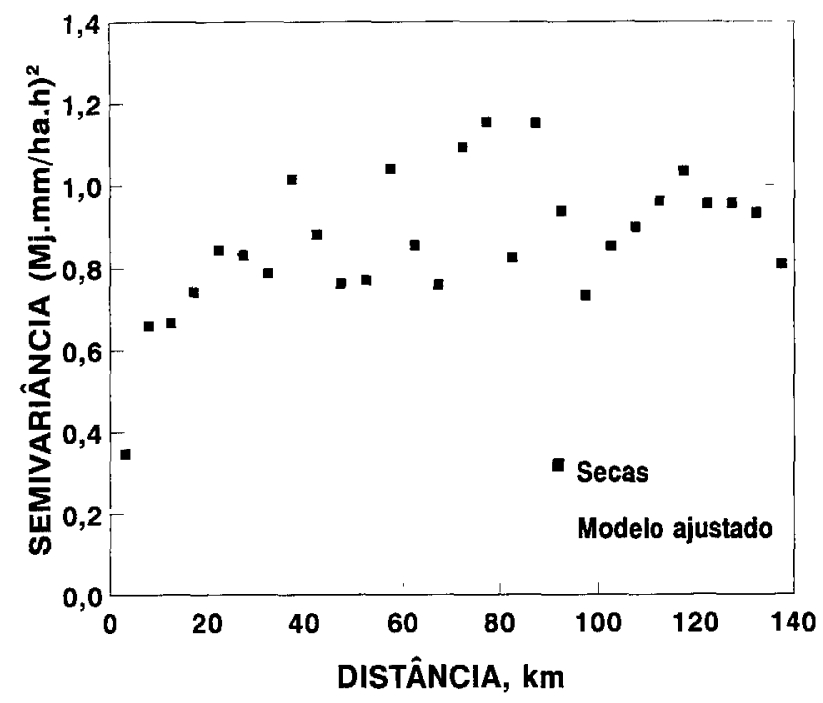

Figura 2. Semivariograma escalonado para a erosividade para o período das secas com modelo exponencial ajustado.

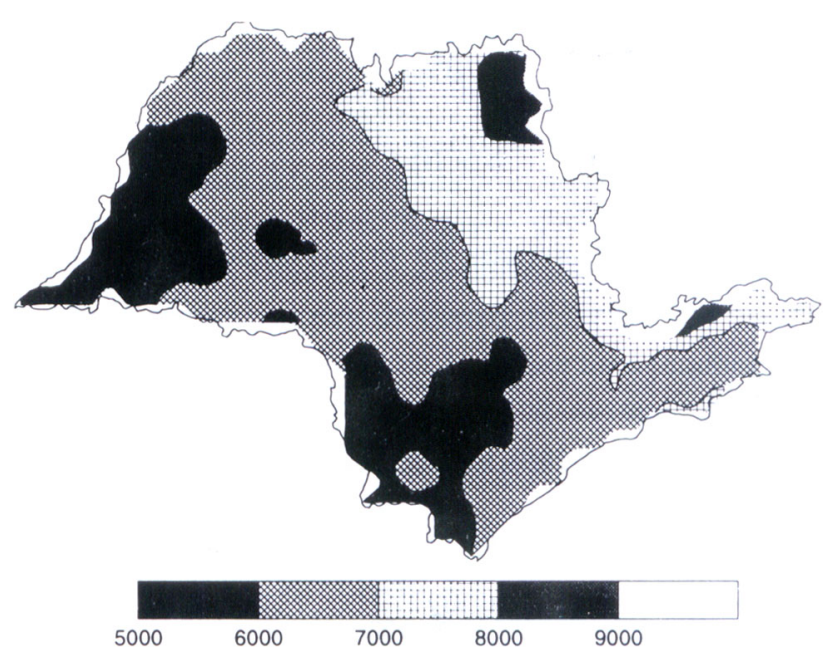

Figura 3. Mapa de isolinhas para erosividade anual 


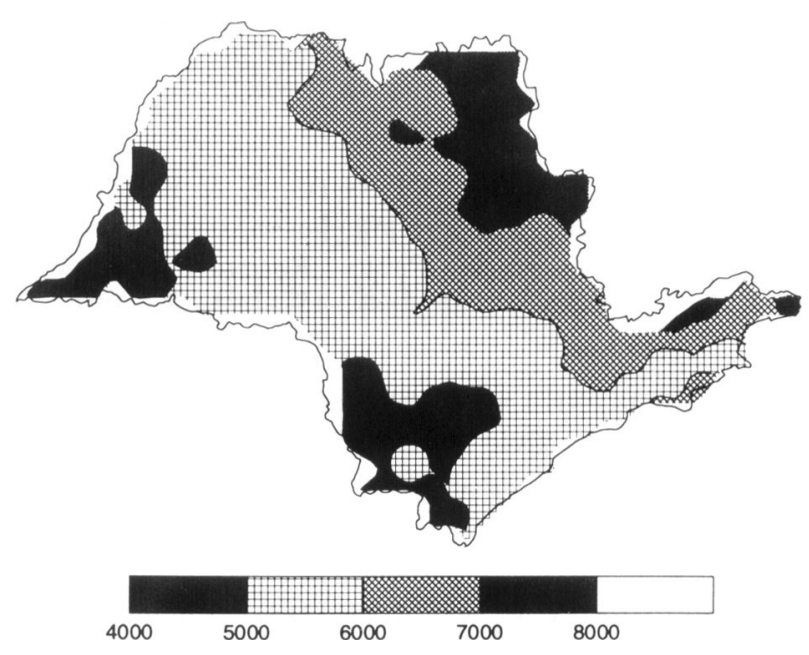

Figura 4. Mapa de isolunhas para crosundade para o período das águas

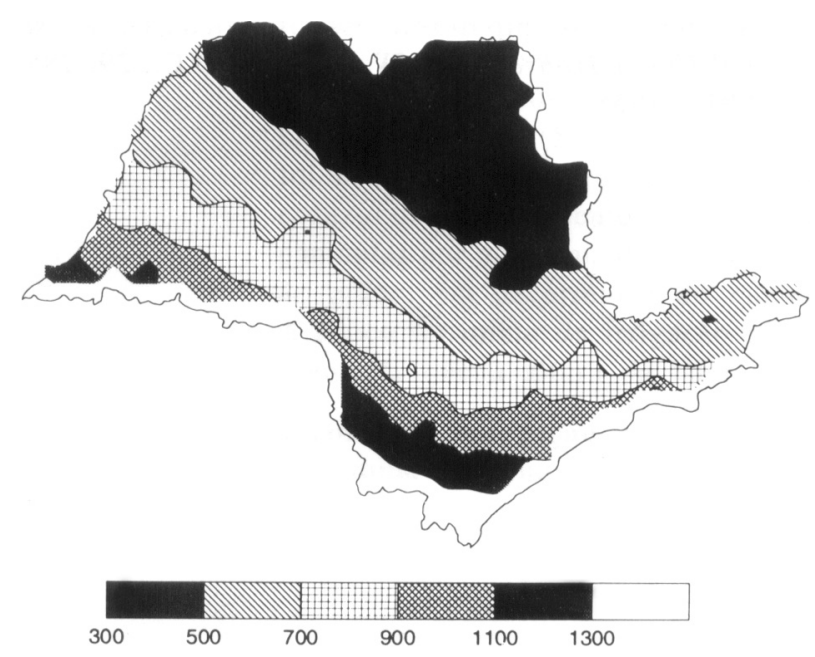

ligura 5. Mapa de isolinhas para crosividade para o período das secas

O mapa de isolinhas para as erosividades correspondentes do período das secas está na figura 5. O paralelismo evidente das isolinhas indica a causa da tendenciosidade encontrada no semivariograma original, razão pela qual se removeu essa tendenciosidade antes da interpolação. As maiores erosividades estão nas regiões Sul e Sudocste do Estado. É de notar também que as regiões com erosividade mínima nas secas, possuem máximas nas águas.
Julgando pelo mapa de isolinhas, que mostrou tendência nítida de aumento das erosividades na direção sul nos meses secos e na direção norte nos chuvosos, é de esperar que a erosividade no Estado de São Paulo não tenha estacionaridade de segunda ordem (Vieira et al., 1983). A remoção da tendenciosidade e o método de auto-validação chamado de "jackknifing" garantem que os modelos dos semivariogramas ajustados representam adequadamente as variabilidades espaciais das erosividades estudadas.O resultado do "jack-knifing" mostrou valores da ordem de 0,001 para o erro relativo médio e 0,93 para as variâncias correspondentes.

\section{CONCLUSÕES}

1. Semivariogramas para o período das águas e para o total anual apresentaram um patamar no valor da variância. O período das secas mostrou tendência de aumentar em alguma direção, necessitando sua remoção, para posterior aplicação de krigagem.

2. Os mapas de isolinhas mostraram que existe aumento quase constante nos valores de erosividade na direção sul nos meses secos e na direção norte nos chuvosos.

3. A geoestatística foi útil na interpretação da variabilidade espacial das erosividades e na obtenção de valores estimados em espaçamentos regulares de $20 \mathrm{~km}$ em todo o Estado para contrução dos mapas de isolinhas.

\section{REFERÊNCIAS BIBLIOGRÁFICAS}

BERTONI, J. \& LOMBARDI NETO, F. Conservação do solo. Ícone, São Paulo, 1990. 355p.

BURGESS, T.M. \& WEBSTER, R. Optimal interpolation and isarithmic mapping of soil properties. I. The semivariogram and punctual kriging. The Journal Soil Science, Oxford, 31:315-331, 1980.

FLANAGAN, D.C., ed. WEPP version 91.5. Water erosion prediction project - Hillslope profile model: documentation, corrections and additions. West Lafayette, USDA-ARS National Soil Erosion Research Laboratory, 1991. 40 p. (NSERL Report, 6) 
HAJRASULIHA, S.; BANIABASSI, H.; METTHEY, J. \& NIELSEN, D.R. Spatial variability of soil sampling for salinity studies in southwest Iran. $I r$ rigation Science, Berlin, 11:197-208, 1980.

KNISEL, W.G., ed. CREAMS: a field-scale model for chemicals, runoff and erosion for agricultural management systems. Washington, United States Department of Agriculture, 1980.640p. (Conservation Research Report, 26)

LOMBARDI NETO, F. \& MOLDENHAUER, W.C. Erosividade da chuva: sua distribuição e relação com as perdas de solo em Campinas (SP). Bragantia, Campinas, 51(2):189-196, 1992.

SĀo PAUlo. Secretaria dos Serviços e Obras Públicas. Departamento de Águas e Energia Elétrica. São Pau10, 1973. 235p. (Boletim pluviométrico, 78)

VIEIRA, S.R.; DE MARIA, I.C.: LOMBARDI NETO, F.; DECHEN, S.C.F. \& CASTRO, O.M. Caracterização da variabilidade espacial de propriedades físicas. In: LOMBARDI NETO, F. \& CAMARGO, O.A. de, coords. Microbacia do Córrego São Joaquim (Município de Pirassununga, SP). Campinas, Instituto Agronômico. 1992. p.41-51. (Documentos IAC. 29)
VIEIRA, S.R.; HATFIELD, J.L.; NIELSEN, D.R. \& BIGGAR, J.W. Geostatistical theory and application to variability of some agronomical properties. Hilgardia, Berkeley, 51(3):1-75, 1983.

VIEIRA, S.R.; LOMBARDI NETO, F. \& BURROWS, I. Mapeamento das precipitações máximas prováveis para o Estado de São Paulo. Revista Brasileira de Ciencia do Solo, Campinas, 15(1):93-98, 1991.

VIEIRA, S.R.; NIELSEN, D.R. \& BIGGAR, J.W. Spatial variability of field measured infiltration rate. Soil Science Society of America Journal, Madison, 45: 1040-1048, 1981.

WISCHMEIER, W.H. \& SMITH, D.D. Predicting raifall erosion losses: a guide to conservation planning. Washington, D.C., United States Department of Agriculture, 1978. 58p. (Agriculture Handbook, 537)

WISCHMEIER, W.H. \& SMITH, D.D. Rainfall energy and its relationship to soil loss. American Geophysical Union Transactions, Washington, D.C., 39: $285-$ $291,1958$. 